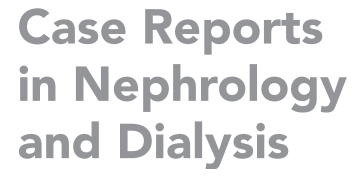

Case Reports and Dialysis

\title{
Recurrence of Cryoglobulinemia Secondary to Hepatitis $C$ in a Patient with HCV RNA (-) Negative in the Serum
}

\author{
Małgorzata Sikorska-Wiśniewska ${ }^{a}$ Katarzyna Sikorska ${ }^{b}$ \\ Anna Wróblewskac Tomasz Liberek ${ }^{a}$ Agnieszka Perkowska-Ptasińskad \\ Alicja Dębska-Ślizieńa \\ aDepartment of Nephrology, Transplantology and Internal Medicine, Medical University \\ of Gdansk, Gdansk, Poland; 'bepartment of Tropical Medicine and Epidemiology, \\ Medical University of Gdansk, Gdansk, Poland; 'Department of Molecular Diagnostics, \\ Intercollegiate Faculty of Biotechnology, University of Gdansk, Gdansk, Poland; \\ ${ }^{\mathrm{d}}$ Department of Transplantation Medicine and Nephrology, Transplantation Institute, \\ Medical University of Warsaw, Warsaw, Poland
}

\section{Keywords}

Membranoproliferative glomerulonephritis - Mixed cryoglobulinemia - Extrahepatic HCV manifestations

\begin{abstract}
Hepatitis $C$ virus infection is associated with many extrahepatic manifestations such as mixed cryoglobulinemia (MC). Renal manifestation of HCV infection might present as cryo-positive membranoproliferative glomerulonephritis (MPGN). First-line therapy includes antiviral treatment as the underlying infection leads to formation of immune complexes. After introducing direct-acting antiviral agents (DAAs) cure rates of HCV infection increased. Sustained virologic response (SVR) is defined as the absence of HCV RNA in serum by a sensitive test performed 12 or 24 weeks after the end of antiviral treatment. Although HCV RNA is undetectable in the serum, it may be present in hepatocytes and peripheral blood mononuclear cells (occult HCV infection). However, the impact of DAA treatment on occult HCV infection is not clear. We report a case of recurrence of MC with MPGN and development of lymphoproliferative disorder 2 years after achieving SVR.
\end{abstract}




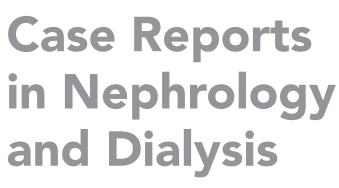

Introduction \begin{tabular}{l|l}
\hline Case Rep Nephrol Dial 2021;11:110-115 \\
\hline DOI: 10.1159/000515587 & $\begin{array}{l}\text { @ 2021 The Author(s). Published by S. Karger AG, Basel } \\
\text { www.karger.com/cnd }\end{array}$ \\
\hline
\end{tabular}

Sikorska-Wiśniewska et al.: Recurrence of Cryoglobulinemia

HCV infection prevalence is estimated for over 70 million adults worldwide [1]. HCV is a major cause of liver cirrhosis, although the virus might affect many organs. Extrahepatic complications of hepatitis $C$ virus infection include immune-related manifestations such as cryoglobulinemia and other lymphoproliferative disorders. The most common renal pathology associated with chronic HCV infection is membranoproliferative glomerulonephritis (MPGN) associated with type II mixed cryoglobulinemia (MC) [2]. Cryoglobulins are immunoglobulins that precipitate at temperature below $37^{\circ} \mathrm{C}$ and dissolve again when heated. $\mathrm{HCV}$ infection is a cause of $70-90 \%$ of MC, which is a systemic vasculitis of small vessels mediated by immune complexes [3]. The pathomechanism of MC involves various mechanisms and includes interaction between the HCV envelope protein E2 and lymphocyte B CD81 receptor, which leads to production of monoclonal IgM with rheumatoid factor (RF) activity. Immune complexes cause vasculitis and comprise viral antigens, immunoglobulins, complement fragments, and cryoglobulins in capillaries, mesangium, and urinary space of glomeruli, which can be manifested as nephritic and nephrotic syndromes [4]. HCV constantly stimulates B cells to proliferate and produce IgM with RF activity, which are deposited in the glomeruli. Clinical manifestations of MC, apart from kidneys, include also skin, joints, and the peripheral nervous system. First-line therapy of $\mathrm{HCV}$-associated MC is an antiviral treatment as the elimination of HCV is crucial to stop immune complex formation and resultant vasculitis. The introduction of direct-acting antiviral agents (DAAs) has increased rates of successful HCV treatment. However, even patients with sustained virologic response (SVR), who have no detectable serum HCV RNA in conventional clinical tests 12-24 weeks after the end of treatment, can still suffer from extrahepatic manifestations of past infection. Moreover, occult HCV infection, which is confirmed by ultrasensitive detection of low-level HCV RNA in the extrahepatic compartment, may have clinical consequences. We present a case report of a patient whose symptoms might have been triggered by an occult HCV infection.

\section{Case Presentation}

In 1998, a 45-year-old female patient presented with abdominal pain, myalgia, and cutaneous vasculitis. Laboratory tests revealed elevated liver enzymes, HCV-antibodies, and positive HCV RNA in the serum. The patient was diagnosed with cryoglobulinemia secondary to HCV infection. Probably she had been infected with HCV during blood transfusions after deliveries in her twenties. Between the years 1999 and 2007, she was unsuccessfully treated for hepatitis C: once using interferon with ribavirin and twice using pegylated interferon with ribavirin. In that time, symptoms were palpable purpura, weakness, neuropathy, arthralgia, dry mouth and dry eyes, and fever. From 2008 to 2013 her well-being improved. Despite positive HCV RNA in the serum, the patient was rather asymptomatic. By this time, she also had not presented any signs of kidney involvement. In February 2014, she was admitted to the nephrology department with nephrotic syndrome and hematuria. Laboratory results included a urinary protein excretion of $7 \mathrm{~g} /$ day, a serum creatinine level of $1.3 \mathrm{mg} / \mathrm{dL}$, a cryocrit of 3.5\%, and cryoglobulin description showed type II MC, monoclonal and polyclonal. During this hospitalization, a percutaneous kidney biopsy revealed MPGN probably associated with cryoglobulinemia. Treatment with steroids wasinitiated with clinical improvement. Four months later, in July 2014, the patient was admitted again to the nephrology department because of acute worsening of renal function. She required emergent hemodialysis, and later, several therapeutic plasma exchanges (TPEs; plasmaphereses) were performed. Therapy with intravenous steroids was continued, followed by oral steroids. In the next few months,

\section{Karger'"}


she was admitted to hospital 3 more times due to fatigue, anemia, and recurrent edema. Further treatment with TPE was continued. The next year, patient's renal function improved. In 2016, she was successfully treated for hepatitis C with DAA (ombitasvir/paritaprevir/ritonavir and dasabuvir) for 12 weeks without severe adverse events. HCV viremia studied by RT-PCR was no longer detectable in the serum, and the patient achieved SVR. In March 2017, she was tested positive for HCV RNA in peripheral blood mononuclear cells (PBMCs) incubated in the presence of mitogens - the test was performed as part of research analysis in patients with extrahepatic manifestations of HCV after successful treatment [5]. HCV RNA in the serum persisted negative. At that moment, she did not present any systemic or renal symptoms suggestive of active HCV infection. In April 2018, the patient was admitted again to the nephrology department with palpable purpura and edema of the lower extremities. We observed nephrotic syndrome with daily protein excretion of $9 \mathrm{~g}$ and a rise in creatinine from $0.85 \mathrm{mg} / \mathrm{dL}$ to $2 \mathrm{mg} / \mathrm{dL}$. She received treatment with intravenous steroids and TPE with moderate effect. HCV RNA in the serum remained undetectable. However, cryoglobulins were present. PBMC analysis was performed again. This time, due to technical reasons, cells were incubated without the presence of mitogens and HCV RNA was undetectable. Due to worsening of kidney function, renal replacement therapy was started. After a few months, diagnostic course revealed hematological disorders: the presence of an abnormal clone of lymphocytes in peripheral blood, which complied with the criteria of monoclonal B-cell lymphocytosis, presence of monoclonal IgM type kappa in serum, and urine immunofixation and abnormal serum-free light chain ratio. Due to nephrotic proteinuria, another percutaneous renal biopsy was performed. The procedure revealed MPGN with immune complexes positive for IgG, IgM, C3, and kappa and lambda light chains localized in mesangium, along GBM and within pseudothrombi in glomerular capillaries (Fig. 1-3). In light of clinical presentation, the overall microscopic picture indicated mixed cryoglobulinemic GN. Chemoimmunotherapy (bendamustine and rituximab) was initiated. Currently, the patient receives rituximab as supportive treatment. Her hematological results improved, and she is no longer dependent on renal replacement therapy.

In order to explain the discrepancy in the results of HCV RNA in PBMC, the analysis was repeated in December 2019, first, without incubation of PBMC in the presence of mitogens and then with the incubation. HCV RNA was positive only in the second part of the analysis. Only positive-strand HCV RNA was present. The negative-strand HCV RNA, which is synthesized during active replication by RNA-dependent RNA polymerase and serves as a template for the production of the positive strand, was absent.

Fig. 1. Cryoglobulinemic GN. Some capillaries are distended and filled with proteinaceous, homogeneous substance (so called "pseudothrombi"). AFOG stain.

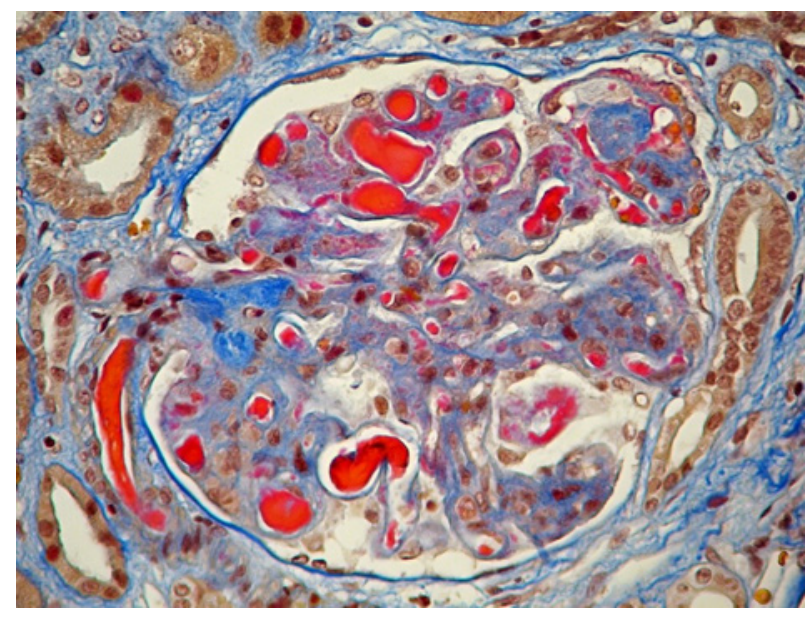




\section{Case Reports in Nephrology and Dialysis}

\begin{tabular}{l|l}
\hline Case Rep Nephrol Dial 2021;11:110-115 \\
\hline DOI: 10.1159/000515587 & $\begin{array}{l}\text { (c) 2021 The Author(s). Published by S. Karger AG, Basel } \\
\text { www.karger.com/cnd }\end{array}$ \\
\hline
\end{tabular}

Fig. 2. Cryoglobulinemic GN. GBM duplication present in some glomerular capillaries (membranoproliferative type of glomerular injury). Silver stain.
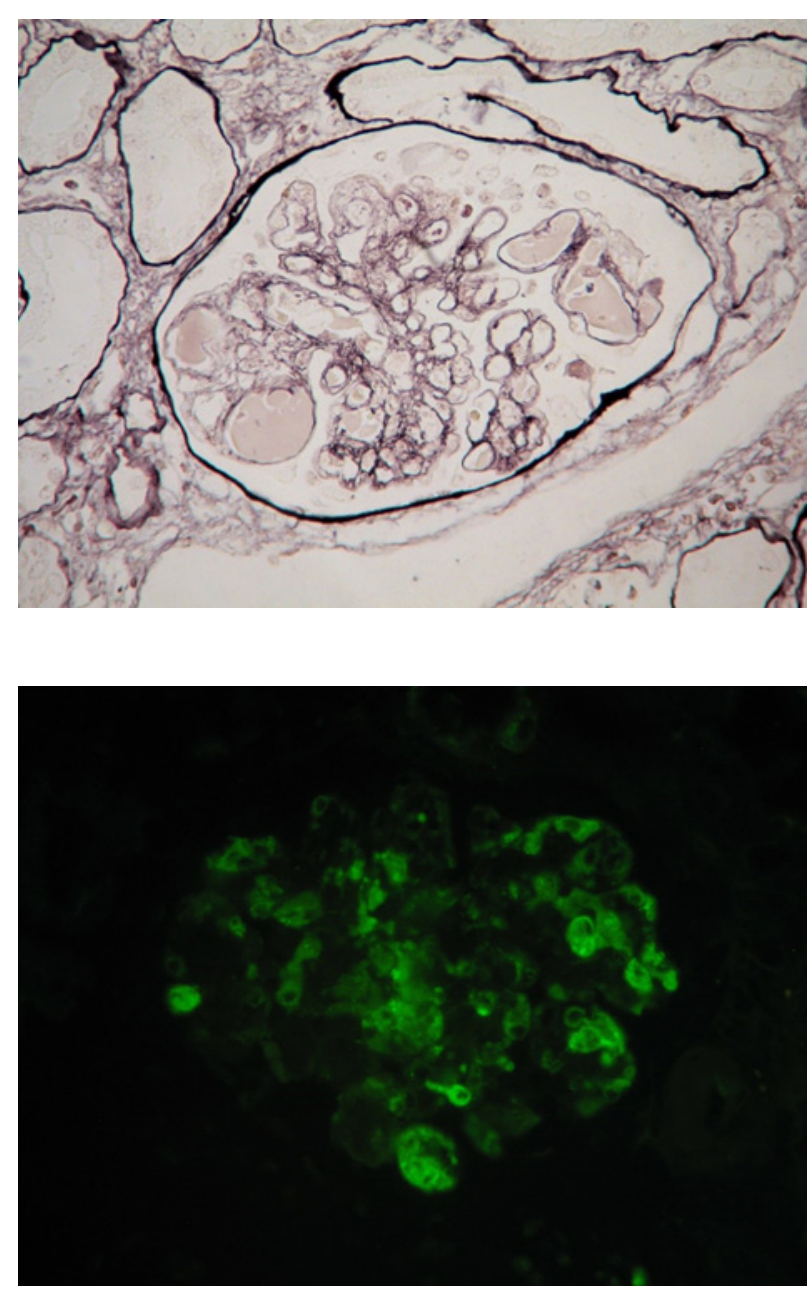

Fig. 3. Cryoglobulinemic GN. Some capillaries are filled with rounded structures that stain brightly for IgM, IgG, and lambda and kappa light chains. Immunofluorescence.

\section{Discussion/Conclusion}

The association between HCV infection and kidney disease is complex, and there are multiple pathways that lead to CKD in infected subjects, including the most common - MPGN. KDIGO guidelines from 2018 recommend HCV screening in all patients at the time of initial evaluation of CKD [6]. Several repetitions of antiviral treatment were unsuccessful in our patient until using DAAs. It is emphasized that antiviral therapies based on IFN and ribavirin have worse outcomes in patients with MC [7]. Managing HCV-associated MC includes immunosuppressive agents, plasma exchanges, and rituximab, which is a B-cell-depleting agent. However, as mentioned above, antiviral treatment is crucial in managing MC. Introduction of DAAs was a breakthrough in treating HCV infection and also in the CKD population as DAAs are better tolerated than IFN and the efficacy is much higher. Symptom recurrence after eradication is not common. To the best or our knowledge, there is only 1 reported case of MC recurrence following the achievement of SVR. In a patient reported by Chowdhury, HCV RNA was undetectable in the serum but present within the cryoprecipitate [8]. The statement that lack of viremia is not tantamount to complete systemic virus eradication was also supported by Castillo et al. [9], who documented intrahepatic HCV RNA in 57\% of individuals who were negative for anti-HCV antibodies and had no HCV RNA detectable in the serum. Furthermore, $70 \%$ of patients with positive intrahepatic HCV RNA were found positive for HCV RNA in PBMCs. 
In the case of MC recurrence reported by us, the patient had detectable HCV RNA only in PBMC and only after incubation in the presence of mitogens. Both tests without mitogens were negative. Diagnosis of occult HCV infection is a possible explanation to the relapse of cryoglobulinemia. Technical issues associated with methodology of detecting occult HCV infections were also discussed and should be taken into account in further studies on clinical consequences of occult HCV infection [10]. Castillo et al. [11] reported a high prevalence of occult HCV infection in patients with primary and secondary glomerulonephritis with no HCV antibodies.

Persistent stimulation of lymphocytes B clones by viral antigens may progress to benign and malignant lymphoproliferative diseases. Monoclonal B-cell lymphocytosis, which is a preclinical condition characterized by expansion of monoclonal B lymphocytes, can be an extrahepatic symptom of occult HCV infection in our patient. Treatment with rituximab as a B-cell-depleting agent seemed to be most accurate in this case, taking into account that symptoms of cryoglobulinemia are most likely caused by lymphocyte proliferation and constant production of IgM RF. The treatment significantly decreased proteinuria and the serum kappa/lambda ratio. Currently, the patient has no clinical symptoms of cryoglobulinemia including skin vasculitis. Due to improvement of renal function, renal replacement therapy was discontinued.

In conclusion, a patient who was previously considered cured from HCV infection developed extrahepatic symptoms. Thorough analysis revealed an occult HCV infection. It should be emphasized that HCV RNA in PBMC was present only after incubating cells in the presence of mitogens. According to Pham et al. [12], incubation with mitogens stimulates proliferation of lymphocytes and increases HCV RNA detection in patients with SVR. Incubation without mitogens seems to be insufficiently sensitive and might lead to underestimation of the actual prevalence of occult HCV infection. Even though introducing DAAs into HCV treatment was a big step forward, there is no evidence that DAAs have influence on residual replication in PBMC.

In this patient, long-term stimulation of lymphocytes B by the virus led to uncontrolled B-cell proliferation and constant production of monoclonal IgM with RF activity. In our opinion, the stimulation was present even after achieving SVR because HCV RNA was still positive in PBMC. It should be taken into account that many diseases such as glomerulonephritis, cryoglobulinemia, and lymphoproliferative disorders may appear years after allegedly cured HCV infection and should be considered as extrahepatic manifestations of occult HCV infection. It is possible that occurrence of this phenomenon is frequent; therefore, PBMC analysis might be a useful tool in the diagnostic process.

\section{Statement of Ethics}

Written informed consent was obtained from the patient for publication of this case report and any accompanying images. Published research complies with the guidelines for human studies and was conducted ethically in accordance with the World Medical Association Declaration of Helsinki. Prof. Alicja Debska-Slizien (Head of the Department of Nephrology, Transplantology, and Internal Medicine of Medical University of Gdansk) approved of the study protocol.

\section{Conflict of Interest Statement}

The authors have no conflicts of interest to declare.

\section{Karger'}




\section{Case Reports in Nephrology and Dialysis}

\begin{tabular}{l|l}
\hline Case Rep Nephrol Dial 2021:11:110-115 \\
\hline DOI: 10.1159/000515587 & $\begin{array}{l}\text { @ 2021 The Author(s). Published by S. Karger AG, Basel } \\
\text { www.karger.com/cnd }\end{array}$ \\
\hline
\end{tabular}

Sikorska-Wiśniewska et al.: Recurrence of Cryoglobulinemia

\section{Funding Sources}

No funding was received for this study.

\section{Author Contributions}

Małgorzata Sikorska-Wiśniewska, Katarzyna Sikorska, and Alicja Dębska-Ślizień wrote the paper. Anna Wróblewska performed biochemical analysis. Tomasz Liberek contributed data. Agnieszka Perkowska-Ptasińska performed the biopsy analysis.

\section{References}

1 World Health Organization. Global hepatitis report 2017. World Health Organization; 2017.

2 Terrier B, Cacoub P. Renal involvement in HCV-related vasculitis. Clin Res Hepatol Gastroenterol. 2013;37(4): 334-9.

3 Cacoub P, Comarmond C, Domont F, Savey L, Saadoun D. Cryoglobulinemia vasculitis. Am J Med. 2015;128(9): 950-5.

4 Fabrizi F, Plaisier E, Saadoun D, Martin P, Messa P, Cacoub P. Hepatitis C virus infection, mixed cryoglobulinemia, and kidney disease. Am J Kidney Dis. 2013;61(4):623-37.

5 Wróblewska A, Lorenc B, Cheba M, Bielawski KP, Sikorska K. Neutrocyte-to-lymphocyte ratio predicts the presence of a replicative hepatitis $\mathrm{C}$ virus strand after therapy with direct-acting antivirals. Clin Exp Med. 2019;19(3):401-6.

6 Kidney Disease: Improving Global Outcomes (KDIGO) Hepatitis C Work Group. KDIGO 2018 clinical practice guideline for the prevention, diagnosis, evaluation, and treatment of Hepatitis C in chronic kidney disease. Kidney Int Suppl (2011). 2018;8(3):91-165.

7 Mazzaro C, Zorat F, Caizzi M, Donada C, Di Gennaro G, Maso LD, et al. Treatment with peg-interferon alfa-2b and ribavirin of hepatitis $C$ virus-associated mixed cryoglobulinemia: a pilot study. J Hepatol. 2005;42(5): 632-8.

8 Chowdhury R, Tsen A. Recurrent mixed cryoglobulinemia despite sustained virologic response to treatment: a case report. Am J Kidney Dis. 2017;70(2):301-4.

9 Castillo I, Pardo M, Bartolomé J, Ortiz-Movilla N, Rodríguez-Iñigo E, de Lucas S, et al. Occult hepatitis C virus infection in patients in whom the etiology of persistently abnormal results of liver-function tests is unknown. J Infect Dis. 2004;189(1):7-14.

10 Pham TN, Michalak TI. Occult hepatitis C virus infection and its relevance in clinical practice.J Clin Exp Hepatol. 2011;1(3):185-9.

11 Castillo I, Martinez-Ara J, Olea T, Bartolomé J, Madero R, Hernández E, et al. High prevalence of occult hepatitis $\mathrm{C}$ virus infection in patients with primary and secondary glomerular nephropathies. Kidney Int. 2014;86(3): 619-24.

12 Pham TNQ, MacParland SA, Coffin CS, Lee SS, Bursey FR, Michalak TI. Mitogen-induced upregulation of hepatitis C virus expression in human lymphoid cells. J Gen Virol. 2005;86(Pt 3):657-66. 